\title{
Human rabies deaths in Africa: breaking the cycle of indifference
}

Betty Dodet ${ }^{\mathrm{a}, *}$, Mathurin C. Tejiokem ${ }^{\mathrm{b}}$, Abdou-Rahman Aguemon ${ }^{\mathrm{c}}$, Hervé Bourhy ${ }^{\mathrm{d}}$ for AfroREB $^{\mathrm{e}}$

${ }^{\text {a }}$ AfroREB coordinator, Dodet Bioscience, 6B rue de Verdun, 69300 Caluire et Cuire, France (B Dodet $\mathrm{PhD}-{ }^{*}$ Corresponding author: Tel: +33472411705 ; E-mail: betty.dodet@dodetbioscience.com)

${ }^{\mathrm{b}}$ Epidemiology and Public Health Service, Centre Pasteur du Cameroun, Yaoundé, Cameroon (MC Tejiokem MD, PhD)

${ }^{\mathrm{c}}$ Faculté des Sciences de la Santé, 01 BP 188, Cotonou, Bénin (Prof AR Aguemon PhD)

${ }^{\mathrm{d}}$ Institut Pasteur, Unité Dynamique des lyssavirus et adaptation à l'hôte, WHO Collaborating Centre for Reference and Research on Rabies, Paris, France (H Bourhy, DVM, PhD)

${ }^{\mathrm{e}}$ The Africa Rabies Expert Bureau (AfroREB), founded in 2008, is an informal network of rabies experts from 15 francophone countries of North and sub-Saharan Africa: Algeria, Morocco, Tunisia, Benin, Burkina Faso, Cameroon, Central African Republic, Congo, Côte d'Ivoire, Gabon, Madagascar, Mali, Niger, Senegal, Togo. 


\begin{abstract}
:
The current outbreak of Ebola virus disease has mobilized the international community against this deadly disease. However, rabies, another deadly disease, is greatly affecting the African continent, with an estimated 25000 human deaths every year. And yet, the disease can be prevented by a vaccine, if necessary with immunoglobulin, even when administered after exposure to the rabies virus. Rabies victims die because of neglect and ignorance, because they are not aware of these life-saving biologicals, or because they cannot access them or do not have the money to pay for them. Breaking the cycle of indifference of rabies deaths in humans in Africa should be a priority of governments, international organizations and all stakeholders.
\end{abstract}

Keywords: Ebola, rabies, Africa 
Since the discovery in 1976 of Ebola virus disease (EVD, formerly known as Ebola hemorrhagic fever), outbreaks of the virus have episodically deserved front page coverage. This was the case in 1995, when an outbreak in the Democratic Republic of Congo killed 250 people, and in 2000-2001 when an outbreak took the lives of 224 people in Uganda. Totally, 1548 EVD deaths out of 2361 cases occurred in Sub-Saharan Africa between 1976 and 2013. The current outbreak in Guinea, Sierra Leone and Liberia, the most devastating yet, may end up with more than 3000 victims. With a case fatality rate up to $90 \%$, EVD is one of the world's most deadly diseases $[1,2]$.

The international engagement against EVD, coordinated by the World Health Organization (WHO) is a good example of how the different stakeholders can mobilize their resources to help the African continent. Many experts have been deployed to help with infection control measures in clinics hospitals, and to trace known contacts of infected patients in the different countries involved. Indeed, in the absence of a vaccine and specific treatment, the only way to control the infection is through protective measures in clinics and hospitals, at community gatherings or at home.

Rabies, another one of the world's most deadly diseases, is also a huge health problem for Africa. Once the first symptoms occur, there is no effective treatment, and the death rate is approaching $100 \%$. Each year, in Africa, rabies kills an estimated 25,000 people, with about one death every 20 minutes. Children are the most affected by the disease, with 4 out of every 10 deaths occurring in children under the age of 15 [3].

In contrast to EVD, rabies is a vaccine-preventable disease $[3,4]$. It can be prevented through timely immunization even after exposure to the deadly virus. There are also effective vaccines for dogs, the main vector and transmitter of rabies to humans. Mass vaccination of dogs is recognized as the most cost-effective and sustainable way to eliminate rabies in humans $[5,6]$.

Why is it that there are still so many deaths from rabies in Africa? This issue was addressed by rabies experts of the public health and veterinary sectors from francophone Africa, during the Fourth AfroREB (Africa Rabies Expert Bureau) meeting that took place in Dakar in October 2013. Established in 2008, AfroREB brings together rabies experts from 15 francophone countries in Africa [7,8].

Like EVD, rabies is linked to poverty, poor health systems and lack of education. The population is often not aware of the rabies risk, and of what to do in case of a dog bite. SubSaharan Africa lacks rabies prevention centers, where bite victims can find the life-saving biologicals (vaccine and immunoglobulin). Health care centers equipped for rabies prevention are scarce and limited to capital cities. They are often not accessible to the rural population, and the biologicals for rabies prophylaxis may not be available or affordable for bite victims. Furthermore, dog rabies remains enzootic in much of the world and attempts to control dog rabies in Africa are either inexistent or not successful, this being largely due to a lack of intersectoral collaboration between ministries, and the considerable challenge posed by the integration of budgets across ministries. 
Therefore, and in contrast to EVD, rabies is trapped in the vicious cycle of indifference. It does not attract media or political attention, as it is one of the oldest recognized diseases and has been controlled in the developed world. As a result of poor surveillance and reporting of rabies cases, there is little reliable data on the rabies burden in African countries [9]. Rabies is frequently misdiagnosed, as it often develops with a variety of symptoms that mimic other encephalitic diseases including cerebral malaria [6]. Cases that are clinically diagnosed are often not reported, thus they are not accounted for at the central level, even in countries where rabies is a notifiable disease. Collecting and shipping skin biopsy samples, or the three serial daily saliva samples, to the laboratory for ante mortem diagnostic confirmation is also an issue, and postmortem diagnosis of brain samples is usually not accepted by the family of the patient or even proposed by physicians [10].

But as long as rabies cases are not reported, rabies deaths will be ignored and the disease will not receive the attention it deserves. Rabies is in competition with many other problems in African countries. With limited resources, and in the absence of awareness of the problem in the population as well as in governments, the priority goes elsewhere. Without resources for sensitization, rabies surveillance or healthcare for bite victims, the number of rabies deaths is increasing. An effective surveillance and notification of animal and human rabies cases is crucial so that the authorities of each country can be aware of the real rabies burden, and give it the place it deserves in their public health programs [8].

The best way to prevent rabies in humans is an integrated 'One Health' approach, as promoted by WHO, the World Organisation for animal Health (OIE) and the Food and Agriculture Organization (FAO). It includes the implementation of programs combining: rabies education and awareness; increased access to post-exposure prophylaxis (PEP) in accordance with WHO recommendations; pre-exposure prophylaxis (PrEP) to those at high risk; large-scale dog vaccination programs and responsible dog ownership. Also, there is a need to strengthen the health system and collaboration with others sectors concerned.

In 1983, recognizing the importance of human rabies transmitted by dogs, the governments of Latin America made the political decision to eliminate the disease and placed rabies on their public policy agendas. With support from the Pan American Health Organization (PAHO), they provided appropriate treatment for people potentially at risk of acquiring the disease (pre- and post-exposure prophylaxis), mass vaccination of dogs, and epidemiological surveillance. Since 1983, Latin America has successfully reduced by more than $90 \%$ canine and human rabies $[4,11]$.

In 2007, the Philippines approved the Anti-Rabies Act into law, that required effective reporting of human and animal rabies, and established a National Rabies Prevention and Control Program based on multisectoral cooperation between the different ministries: (Agriculture, Health, Education) and the Local Government Units, with the assistance of international organizations (WHO, OIE) and NGOs (Global Alliance for Rabies ControlGARC). The program includes: education campaigns, increased access to PEP by the establishment of animal bite prevention centres all over the country, pre-exposure prophylaxis 
for children in areas where rabies incidence exceeds 2.5 human rabies cases per million population, and mass vaccination of dogs [12]. The number of human rabies cases has declined by $22 \%$ from $2005-2012$.

Currently, pilot projects that aim to eliminate dog rabies are being conducted in Africa and include a project coordinated by WHO and funded by the Bill \& Melinda Gates Foundation in KwaZulu Natal (South Africa) and in Tanzania, and a program carried out in N'Djamena (Chad) by the Chad government, the Swiss Tropical and Public Health Institute and GARC. Sub-regional networks of African rabies experts, SEARG (Southern and Eastern African Rabies Group) and AfroREB are advocating for rabies control, in line with the Global Alliance for Rabies Control. Several North-South initiatives and networks for rabies control in Africa are being established, such as ICONZ (Integrated Control of Neglected Zoonoses, www.iconzafrica.net), and ADVANZ (Advocacy for Neglected Zoonotic Diseases, www.advanz.org). Both of these programs aim to control neglected zoonotic diseases, including rabies. RESOLAB initially established by FAO to strengthen the African Veterinary laboratories diagnostic capacities for avian flu, now includes some support for rabies diagnosis. The International Pasteur Institute Network plays an important role in rabies diagnostic, prevention and control as well as in capacity building in the field of rabies over the world, including in Africa [13,14]. These efforts should be pursued and coordinated in order to reduce the rabies burden in Africa.

It is not the objective of this Commentary to attempt to define which disease, EVD or rabies, is most deadly and deserves the most attention, as both are urgent public health concerns. As tools do exist that could prevent the estimated 25,000 human rabies deaths every year in Africa, it is a shame not to use them or to more actively promote their use.

AfroREB members recognized that breaking the cycle of indifference is the first priority, and they call on their governments, international organizations and all stakeholders to give rabies the attention it deserves, and to unite their efforts to promote the elimination of dogtransmitted rabies in Africa.

\section{Acknowledgements}

The authors would like to thank Dr Deborah Briggs, past-Executive Director, Global Alliance for Rabies Control, for her advice and support.

AfroREB benefits from a grant from Sanofi Pasteur and Merial.

\section{References}

1. World Health Organization. Global Alert and Response (GAR). Ebola virus disease. http://www.who.int/csr/disease/ebola/en/ [Accessed 14.08.2014].

2. Centers for Disease Control and Prevention. Ebola Hemorrhagic Fever. Chronology of Ebola Hemorrhagic Fever Outbreaks. http://www.cdc.gov/vhf/ebola/resources/outbreak-table.html [Accessed 22.07.2014] 
3. World Health Organization. Rabies. http://www.who.int/rabies/en/ [Accessed 14 August 2014]

4. World Health Organization. WHO Expert consultation on rabies. Second Report. WHO Technical Report Series 982, 2013.

5. Lembo T, Partners for Rabies Prevention. The blueprint for rabies prevention and control: a novel operational toolkit for rabies elimination. PLoS Negl Trop Dis 2012;6(2):e1388.

6. Fooks AR, Banyard AC, Horton DL, Johnson N, McElhinney LM, Jackson AC. Current status of rabies and prospects for elimination. Lancet 2014; May 9. pii: S01406736(13)62707-5. doi: 10.1016/S0140-6736(13)62707-5. [Epub ahead of print].

7. Dodet B, Africa Rabies Expert Bureau (AfroREB). Fighting rabies in Africa: the Africa Rabies Expert Bureau. Vaccine 2008;26(50):6295-8.

8. Dodet B, Africa Rabies Bureau (AfroREB). The fight against rabies in Africa: From recognition to action. Vaccine 2009;27(37):5027-32.

9. Nel LH. Discrepancies in data reporting for rabies, Africa. Emerg Infect Dis 2013;19(4):529-33.

10. Dacheux L, Wacharapluesadee S, Hemachudha T, Meslin FX, Buchy P, Reynes JM, Bourhy $\mathrm{H}$. More accurate insight into the incidence of human rabies in developing countries through validated laboratory techniques. PLoS Negl Trop Di 2010;4(11):e765.

11. Schneider MC, Belotto A, Paz Adé M, Hendrickx S, Leanes LF, de Freitas Rodrigues MJ, Medina G. Current status of human rabies transmitted by dogs in Latin America. Cad. Saude Publica, Rio de Janeiro 2007;23(9):2049-63.

12. Quiambao BP. Rabies pre-exposure prophylaxis. In: 15th Pediatric Infectious Disease Society of the Philippines (PIDSP) Annual Convention. 2008. http://www.pidsphil.org/pdfviewer.php?id=15 [accessed 28.08.2014].

13. Bourhy H, Dautry-Varsat A, Hotez PJ, Salomon J. Rabies, still neglected after 125 years of vaccination. PLoS Negl Trop Dis 2010;4(11): e839.

14. Workshop on Surveillance and Control of Rabies - Pasteur institute Dakar December 3-14, 2013. http://predemics.biomedtrain.eu/cms/Default.aspx ?Page=19812\%20\&menu=494 